\title{
Competencias docentes del joven profesor de educación física de segunda enseñanza
}

\author{
Teaching competences of the young teacher of physical \\ education of secondary education
}

\author{
Phd, Rubén Castillejo Olán \\ ruben.castillejoo@ug.edu.ec.
}

Recibido: 1/12/2017, Aceptado: 1/02/2018

\begin{abstract}
RESUMEN
El perfil de competencias del profesor de Educación Física que labora en el nivel medio de educación, en su etapa de inducción profesional, está sustentado un modelo que expresa el saber, el saber - hacer, y el saber - ser de su actividad laboral; en su construcción se emplearon los métodos del enfoque sistémico y la modelación; así como el análisis de documentos, las encuestas, observación y entrevistas. Este perfil está conformado por un conjunto de competencias docentes de naturaleza compleja, a decir: competencia pedagógica-didáctica, competencia físico-recreativa-deportiva y competencia investigativa; así como las descripciones detalladas de las cualidades que han de caracterizar el desempeño de este profesional; y su contenido permite modelar el estado deseado, en su desempeño, lo que se constituye un referente para la determinación del contenido de su crecimiento profesional.
\end{abstract}

Palabras clave: Perfil de competencias, Educación Física, inducción profesional, competencias docentes, profesor novel

\begin{abstract}
The profile of the competences of the Physical Education teacher working in the middle level of education, in his stage of professional induction, is supported by a model that expresses the knowledge, know - how, and know - how of his work activity; In its construction the methods of the systemic approach and the modeling were used; As well as document analysis, surveys, observation and interviews. This profile is made up of a set of teaching competences of a complex nature, namely: pedagogical-didactic competence, physical-recreational-sports competence and investigative competence; As well as the detailed descriptions of the qualities that must characterize the performance of this professional; And its content allows modeling the desired state in its performance, which constitutes a reference for determining the content of its professional growth.
\end{abstract}

Keywords: Competence profile, Physical Education, professional induction, teacher competencies, novel teacher

$\overline{1}$ Docente y Gestor General de Acreditación de la Universidad de Guayaquil. Ecuador 
ब बt

No. 18,30 de abril de 2018

ISSN impreso: 1390 - 6321

\section{Introducción}

Diseñar un perfil de competencias para una determinada categoría de profesionales, es un proceso que ha de tener entre sus fuentes más importantes las exigencias de su actividad profesional (Chiavenato, 1996; Cuesta, 2001). La actividad profesional del profesor de educación física es una actividad de tipo docente, por lo tanto, estaremos haciendo referencia a competencias docentes, según Blázquez:

La competencia docente es un término que hace referencia a la capacidad del profesorado para intervenir de un modo eficaz en el proceso de enseñanza aprendizaje, es el conjunto de aquellas experiencias, comportamientos, conocimientos y actitudes que pueden definir al docente dentro de la enseñanza de la educación física. (Blázquez, 2013, p.13)

El desarrollo profesional del docente ha sido investigado por diferentes autores, todos coinciden en señalar que ese desarrollo transita por etapas; según Villar (1990) se manifiesta en tres etapas: la inducción, la renovación y la redirección, e Imbernón (1994) considera que existe una primera etapa de formación básica y socialización profesional, una segunda de inducción profesional y socialización en la práctica y una tercera, que denominó etapa de perfeccionamiento. Los autores coinciden en identificar una etapa que se ubica posterior al egreso de la formación inicial, que se caracteriza por el inicio de la vida profesional del docente, a la que se denomina inducción profesional.

En la inducción profesional se manifiestan una serie de insuficiencias debido a que necesitan madurar en la práctica educativa; la docencia en educación física no es una excepción; en tal sentido se planteó como problema científico: ¿cómo identificar las competencias docentes del profesor de educación física de educación media para que sirvan de patrón referencial en el desarrollo de sus acciones de superación profesional?

Son muchos los investigadores que han incursionado en los problemas de los profesores noveles en la etapa de inducción profesional; destaca la investigación de Veenman (1984), que incluyó muestras de jóvenes docentes de Estados Unidos, Alemania, Inglaterra, Holanda, Australia, Canadá, Austria, Suiza y Finlandia; Vera (1988), Granda (1988), Villar (1990), Vonk (1996), Siedentop (1998), Sáenz, López, Castillo y Sáez (2009); González (2013) y Goncalves, Lemos, Correa y Toro (2014), todos ellos identificaron una serie de problemas que resultan comunes para los docentes principiantes, y reconocen la necesidad de programas de inducción para su solución; sin embargo las mayores limitaciones radican en el cómo identificar los contenidos de ese programa para que encuentren una armonía entre el currículo de tercer nivel cursado, la formación básica y la especialización, con particularidad en las necesidades educativas para su superación.

El perfil de competencias docentes se construyó sobre la base de un modelo (Castillejo, 2004), que tiene trece (13) premisas teóricas y empíricas, que surgen del estudio de sistematización teórica y de las mejores experiencias investigativas y laborales; así como es contentivo de cuatro (4) componentes fundamentales, que son: elaboración del perfil de competencias docentes, el diagnóstico de las necesidades educativas del joven graduado, diseño y aplicación del programa de inducción y la 


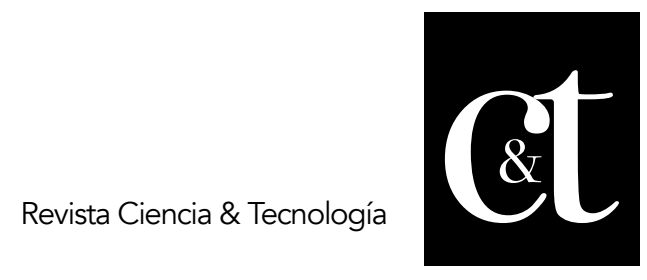

No. 18, 30 de abril de 2018

ISSN impreso: 1390 - 6321

evaluación del impacto del programa inductivo.

\section{Métodos}

La muestra que empleó la investigación estuvo compuesta por once (11) egresados de la carrera de licenciatura en cultura física de la Universidad de Ciencias de la Cultura Física y el Deporte "Manuel Fajardo", en su Facultad de Holguín, que constituye el $100 \%$ de los profesores de educación física en la educación media, en esa etapa. Fueron encuestados veinte (20) profesores de educación física del nivel, de los centros donde se desempeñaban los profesores noveles, y quince (15) gestores de diferentes niveles. Los métodos de modelación y el enfoque sistémico se utilizaron en la concreción del modelo de competencias docentes del profesor de educación física de educación media en inducción profesional; el análisis - síntesis y la inducción - deducción, para la valoración de la información de fuentes teóricas. Además, fueron empleados la observación, el análisis de documentos y el criterio de expertos; la encuesta y la entrevista.

Los métodos estadísticos fueron utilizados para el procesamiento de la información obtenida de diferentes fuentes.

\section{Metodología empleada}

La elaboración del perfil de competencias docentes se realizó por la siguiente metodología (Castillejo 2004), la que transitó por tres fases:

1. Determinación de las principales exigencias a la actividad profesional del docente de educación física durante su etapa de inducción profesional.

2. Identificación de las competencias docentes y las cualidades que revelan su nivel de desarrollo.

3. Valoración teórica del perfil de competencias docentes identificadas. Las acciones en deben conducir a:

a). - Determinar los requerimientos del puesto de trabajo del profesor de educación física de segunda enseñanza. Se deben identificar las funciones, los deberes y las responsabilidades, pueden también obtenerse de fuentes personales a través de la aplicación de métodos y técnicas de investigación.

b). - Análisis del modelo del profesional de la formación de tercer nivel. Permite identificar los objetivos de la carrera de licenciatura en educación física, deportes y recreación declarados en el modelo del profesional, que están expresados en forma de habilidades o competencias, con énfasis en los que se refieren a la educación física.

c). - Análisis de las prioridades del trabajo de la segunda enseñanza, de los objetivos de la formación del escolar en el nivel y el papel que le corresponde a la educación física en su alcance. Parte del estudio de las prioridades del trabajo metodológico de la enseñanza y de los objetivos formativos de los diferentes niveles y grados escolares, permite identificar requerimientos a considerar en el proceso didáctico de la clase de educación física y las implicaciones en la gestión docente.

d). - Análisis de las prioridades del trabajo metodológico de la educación física para el período de trabajo y de las implicaciones de las concepciones de su didáctica para 


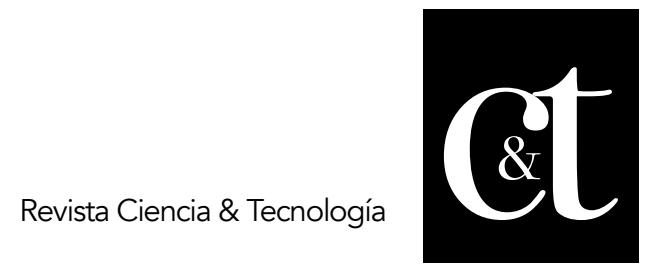

No. 18, 30 de abril de 2018

ISSN impreso: 1390 - 6321

el desempeño de los jóvenes docentes. Para cumplir con este propósito pueden emplearse los documentos que conforman los modelos de formación en las diferentes enseñanzas, documentos normativos e indicaciones para el desarrollo de la educación física, y de fuentes bibliográficas relacionadas con el tema. Una vez identificadas las competencias docentes debe procederse a la determinación de su pertinencia mediante métodos científicos.

\section{Resultados y discusión}

Las exigencias a la actividad profesional del profesor de educación física de segunda enseñanza están referidas a:

$\checkmark$ La participación en el trabajo formativo del colectivo pedagógico, lo que tiene, entre otras, las siguientes implicaciones: la promoción de la práctica sistemática de actividad física en el alcance de una cultura motriz, la organización y ejecución de actividades formativas extradocentes y extraescolares, de conjunto con el colectivo pedagógico el diseño, organización, ejecución, control y evaluación de las estrategias educativas, el desarrollo de acciones de orientación profesional y formación vocacional hacia la actividad física, y los valores que ha de portar como educador.

$\checkmark$ La gestión del proceso de enseñanza - aprendizaje de la educación física. Presupone la programación, organización, desarrollo y la evaluación, el desarrollo del trabajo formativo escolar, la aplicación de las tecnologías de la Información y las comunicaciones en la gestión docente, el cuidado, creación y reparación de los materiales docentes.

$\checkmark$ La gestión deportiva escolar y los festivales físico-recreativos, lo que implica la preparación de equipos deportivos representativos de la institución para los juegos intra y extraescolares, la realización de festivales deportivos - recreativos, y el desarrollo de actividades recreativas en la naturaleza.

$\checkmark$ La participación en actividades de desarrollo profesional, tales como: los concursos de clases de educación física, el desarrollo de acciones investigativas para solucionar problemas profesionales y la divulgación de sus resultados, la participación en las acciones de trabajo metodológico y de capacitación.

Competencias docentes del novel profesor de educación física de segunda enseñanza en su etapa de inducción profesional.

Los estudios realizados en diferentes contextos acerca de la inducción profesional como primera etapa del desarrollo profesional de los docentes, sugieren que las exigencias al desempeño profesional de los recién graduados, en este período, deben ser planteadas en un nivel primario o básico, lo que no niega que tales exigencias sean contentivas de metas retadoras (desarrolladoras) que propicien, a partir del diagnóstico de partida y la ayuda oportuna de conductores y gestores, el desarrollo profesional y la creación de condiciones para su continuidad una vez concluido este período (Veenman, 1984; Vera, 1988; Villar, 1990; Imbernon, 1994).

Como resultado del proceso investigativo se identificaron tres competencias fundamentales, que son: competencia pedagógica-didáctica, competencia físicorecreativa-deportiva y competencia investigativa. 


\section{Competencia pedagógica-didáctica}

El contenido esencial de esta competencia se revela a través de la conjugación armónica en el desempeño profesional de los conocimientos, habilidades, capacidades, actitudes y valores necesarios para la gestión del proceso formativo de adolescentes y jóvenes, desde las particularidades de la actividad física, sustentado en las concepciones de la didáctica de la educación física. Se considera que el recién graduado es portador de esta competencia cuando demuestra en su desempeño profesional:

1. Conocer el fin y los objetivos de la enseñanza en que labora.

2. Conocer las características psicológicas, biológicas, sociales y motrices de los escolares de segunda enseñanza.

3. Conocer las particularidades de los métodos de trabajo educativo con los adolescentes y jóvenes.

4. Saber utilizar las técnicas de cómputo y las comunicaciones al proceso de enseñanza - aprendizaje.

5. Saber promover la práctica sistemática de actividades físicas.

6. Conocer los objetivos y el contenido de los programas de educación física de segunda enseñanza.

7. Saber derivar, elaborar y orientar los objetivos en la clase.

8. Saber planear y desarrollar el estudio diagnóstico.

9. Saber seleccionar los ejercicios y dosificar el trabajo en correspondencia con los objetivos, la edad, el sexo, desarrollo físico, experiencia motriz, y desarrollo cognitivo.

10. Saber emplear estrategias de aprendizaje el proceso de enseñanza - aprendizaje.

11. Saber organizar a los estudiantes en correspondencia con los materiales, las estrategias, la cantidad y el espacio.

12. Saber planificar, observar y evaluar el desarrollo de los escolares.

13. Saber comunicarse adecuadamente con los estudiantes durante la gestión didáctica.

14. Ser responsable y mostrar una conducta adecuada en su actividad profesional.

Competencia físico-recreativa-deportiva:

La educación física en la segunda enseñanza emplea como medios los juegos, deportes, gimnasia, lo que precisa de una serie de requerimientos específicos relacionados con su dominio técnico-profesional. El contenido de esta competencia se revela a través de: 


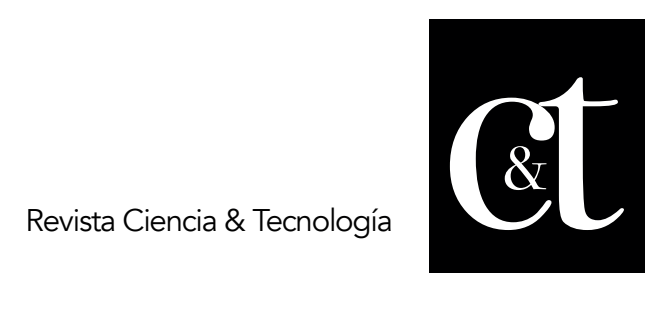

No. 18, 30 de abril de 2018

ISSN impreso: 1390 - 6321

1. Saber ejecutar los elementos básicos de la técnica de los deportes incluidos en la malla curricular.

2. Conocer básicamente las reglas y las particularidades del arbitraje de los deportes motivos de estudio.

3. Saber planificar, organizar y desarrollar competencias deportivas en la escuela.

4. Saber planificar y desarrollar la preparación de los equipos deportivos representativos de la escuela.

5. Saber organizar una composición gimnástica y grupos de gimnasia musical aeróbica.

6. Saber programar, organizar y conducir festivales físico-deportivo-recreativos

7. Saber programar, organizar y desarrollar actividades en la naturaleza, desde una visión ecológica y sostenible.

8. Saber cuidar, elaborar y mantener en buen estado los materiales didácticos.

Competencia investigativa:

El profesor de educación física necesita dar solución a problemas profesionales que van surgiendo en su práctica educativa y enfrentarlos de manera reflexiva y crítica, empleando el método científico. El contenido esencial de esta competencia se revela a través de:

1. Saber programar, desarrollar y registrar datos como consecuencia de la aplicación de métodos investigativos, y realizar análisis conclusivo.

2. Saber determinar problemas que requieran del trabajo científico para su solución.

3. Saber aplicar experiencias didácticas de avanzada para la solución de problemas de su práctica profesional.

4. Saber elaborar ponencias para la divulgación de los resultados de su actividad científica.

5. Saber comunicar los resultados de su actividad científica.

6. Saber realizar la gestión documental de sus necesidades didácticas e investigativas.

7. Ser ético en la labor investigativa como expresión del compromiso con la educación física.

Las tablas que se muestran a continuación constituyen resultado de la aplicación del método Delphi, a treinta y uno (31) expertos, de cuarenta y tres (43) posibles, seleccionados mediante la determinación de la coeficiencia de competencia.

Los resultados de la aplicación de una encuesta a dichos expertos permitieron determinar la pertinencia de los cuatro componentes que conforman el modelo teórico 
metodológico, donde se sustenta el perfil de competencias; al observar la tabla IV, se aprecian los puntos de corte, donde $\mathrm{C} 1=$ muy relevante, $\mathrm{C} 2=$ relevante, $\mathrm{C} 3=$ medianamente relevante, $\mathrm{C} 4=$ poco relevante y $\mathrm{C} 5=$ no relevante; evidenciando que los expertos tienen consenso en la calificación de muy relevantes.

Tabla 1. Matriz de Frecuencias Acumuladas

\begin{tabular}{|l|l|l|l|l|l|l|}
\hline Componentes & C1 & C2 & C3 & C4 & C5 & TOTAL \\
\hline Componente 1 & 28 & 2 & 1 & 0 & 0 & 31 \\
\hline Componente 2 & 30 & 1 & 0 & 0 & 0 & 31 \\
\hline Componente 3 & 29 & 2 & 0 & 0 & 0 & 31 \\
\hline Componente 4 & 31 & 0 & 0 & 0 & 0 & 31 \\
\hline
\end{tabular}

Fuente: Elaboración propia

Tabla 2. Matriz de Frecuencias Acumuladas

\begin{tabular}{|l|c|c|c|c|c|}
\hline Componentes & C1 & C2 & C3 & C4 & C5 \\
\hline Componente 1 & 28 & 30 & 31 & 31 & 31 \\
\hline Componente 2 & 30 & 31 & 31 & 31 & 31 \\
\hline Componente 3 & 29 & 31 & 31 & 31 & 31 \\
\hline Componente 4 & 31 & 31 & 31 & 31 & 31 \\
\hline
\end{tabular}

Fuente: Elaboración propia

Tabla 3. Matriz de Frecuencias Relativas (probabilidades) Acumuladas

\begin{tabular}{|l|c|c|c|c|}
\hline Componentes & C1 & C2 & C3 & C4 \\
\hline Componente 1 & 0,9032 & 0,9677 & 1 & 1 \\
\hline Componente 2 & 0,9677 & 1 & 1 & 1 \\
\hline Componente 3 & 0,9355 & 1 & 1 & 1 \\
\hline Componente 4 & 1 & 1 & 1 & 1 \\
\hline
\end{tabular}

Fuente: Elaboración propia 
Tabla 4. Matriz de Valores de Abscisas

\begin{tabular}{|l|c|c|c|c|c|c|}
\hline Componentes & C1 & C2 & C3 & C4 & Promedio & N - Prom. \\
\hline Componente 1 & 1,3 & 1,85 & 3,49 & 3,49 & 2,53 & 0,5 \\
\hline Componente 2 & 1,85 & 3,49 & 3,49 & 3,49 & 3,08 & $-0,05$ \\
\hline Componente 3 & 1,52 & 3,49 & 3,49 & 3,49 & 3 & 0,03 \\
\hline Componente 4 & 3,49 & 3,49 & 3,49 & 3,49 & 3,49 & $-0,46$ \\
\hline Suma & 8,16 & 12,32 & 13,96 & 13,96 & 12,1 & \\
\hline Punto de corte & 2,04 & 3,08 & 3,49 & 3,49 & 3,03 & $=\mathrm{N}$ \\
\hline
\end{tabular}

Fuente: Elaboración propia

\section{Conclusiones}

Las competencias docentes pedagógica-didáctica, físico-recreativa-deportiva e investigativa del joven profesor de educación física de segunda enseñanza durante su inducción profesional, surgidas de las exigencias de su actividad profesional, la sistematización de las mejores experiencias investigativas constituye el principal referente para la determinación de sus principales necesidades educativas.

El perfil de competencias docentes del joven profesor de educación física de segunda enseñanza durante su inducción profesional, permite modelar el proceso de superación y capacitación, para contribuir de manera armónica a su crecimiento profesional y humano.

\section{Referencias bibliográficas}

Blázquez Sánchez, D. (2013). Diez competencias docentes para ser mejor profesor de educación física. La gestión didáctica de la clase. Barcelona, España: Editorial INDE.

Castillejo Olán, R. (2004). La dirección de la superación del profesor de EFE durante el Adiestramiento Laboral (tesis doctoral). ISCF "Manuel Fajardo", La Habana.

Chiavenato, (1996). Administración de Recursos Humanos. México: Editora Mc Graw Hill.

Cuesta Santos, A. (2001). Gestión de competencias. La Habana: Editorial Academia.

Goncalves, L., Lemos, F., Correa, D. y Toro, S. (2014). Formación de profesores en Educación Física en Brasil: comprensión de estudiantes noveles de la Universidad Federal de San Carlos. Estud. pedagóg. vol. 40 no. Especial, Valdivia 2014. Recuperado

https://scielo.conicyt.cl/scielo.php?script=sci arttext\&pid=S0718$\underline{07052014000200006}$

González Calvo, G. (2013). La lucha de un docente novel de educación física por el reconocimiento de su profesión narrada desde una perspectiva autobiográfica. Revista Agora, No. 15 (2), mayo - agosto 2013: 80-95. Recuperado de http://agorarevista.blogs.uva.es/files/2013/11/agora_15_2a_gonzalez.pdf

Granda, J. (1988). Análisis del pensamiento de docentes nóveles en educación física 


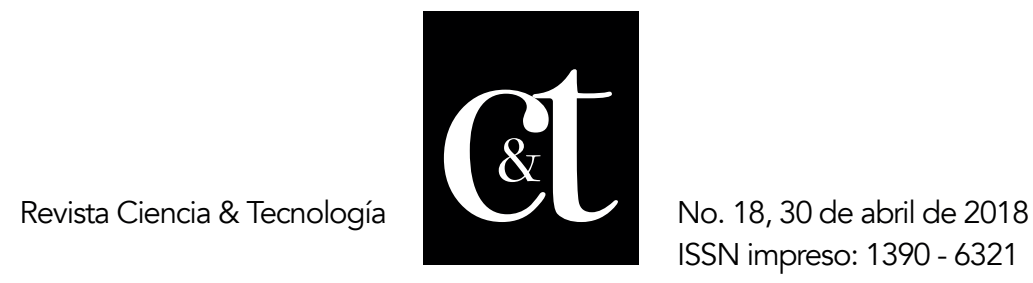

mediante la utilización conjunta de técnicas cuantitativas y cualitativas. Revista Motricidad, 4: 159-181. Recuperado de file://C:/Users/U\%20de\%20Guayaaquil/Downloads/DialnetAnalisisDelPensamie ntoDe DocentesNovelesEnEducacion-2278263.pdf

Imbernon, F. (1994). La formación y desarrollo profesional del profesorado, hacia una nueva cultura profesional. Barcelona: Ed. Graó.

Sáenz, P., López, B., Castillo Viera, E., y Sáez Padilla, J. (2009). Programa de formación para profesores universitarios principiantes de Educación Física. Revista efdeportes. Recuperado de http://www.efdeportes.com/efd134/formacion- paraprofesores-principiantesde-educacion-fisica.htm

Siedentop, D. (1998). Aprender a enseñar la Educación Física. Barcelona: INDE.

Veenman, S. (1984). Perceived problems of beginning teacher. Review of Educational Research, 25(2), 43-178.

Vera, J. (1988). El Profesor principiante. Valencia: Promolibro.

Villar Angulo, L. (1990). El profesor como profesional: formación y desarrollo personal. España: Universidad de Granada.

Vonk, J.H.C. (1996). A Knowledge Base For mentors of Beginning teachers: Result of A Dutch Experience. In: R. McBridge (ed.). Teacher Education Policy. p. 112-134. London: Falmer Press. 